\title{
PHASE TRANSITIONS AND CHANGE OF TYPE IN LOW-TEMPERATURE HEAT PROPAGATION*
}

\author{
KATARZYNA SAXTON ${ }^{\dagger}$ AND RALPH SAXTON ${ }^{\ddagger}$
}

\begin{abstract}
Classical heat pulse experiments have shown heat to propagate in waves through crystalline materials at temperatures close to absolute zero. With increasing temperature, these waves slow down and finally disappear, to be replaced by diffusive heat propagation. Several features surrounding this phenomenon are examined in this work. The model used switches between an internal parameter (or extended thermodynamics) description and a classical (linear or nonlinear) Fourier law setting. This leads to a hyperbolic-parabolic change of type, which allows wavelike features to appear beneath the transition temperature and diffusion above. We examine the region around and immediately below the transition temperature, where dissipative effects are insignificant.
\end{abstract}

Key words. phase transition, hyperbolic-parabolic, change of type

AMS subject classifications. 35M10, 35Q72, 35R35

DOI. $10.1137 / 040612208$

1. Introduction. The analysis in this paper is based on a low-temperature heat propagation model described in [9] and [10]. The model is based on experimental results of [3], [2], [5], and [6], which provide evidence of second sound, i.e., hyperbolic, or wavelike, thermal effects where Fourier's law fails, in very pure crystals of sodium fluoride and bismuth.

Significantly, these features appear only at certain temperatures below which the materials reach their peak thermal conductivities (at approximately $18.5 \mathrm{~K}$ and $4.5 \mathrm{~K}$ for $\mathrm{NaF}$ and $\mathrm{Bi}$, respectively). No wavelike behavior is found in $\mathrm{NaF}$ and $\mathrm{Bi}$ at higher temperatures, where only diffusive heat propagation is observed. Further, the speed, $U_{E}$, at which small amplitude thermal waves propagate is a decreasing function of temperature in the region where the waves can be detected, after which the diffusion process dominates. This hyperbolic region appears separated from the diffusive region by a "critical" temperature, $\vartheta_{\lambda}$, at which $U_{E}=0$ [1]. The aim of this paper is to understand the dynamics of regular solutions having temperatures close to that of the phase transition. We begin, in section 2, by describing a phenomenological onedimensional model which uses an internal variable behaving as an order parameter. In section 3, we will examine properties of the phase transition, and in section 4, we obtain conditions under which this class of solutions remain smooth. Some explicit cases are, finally, examined in section 5 .

2. Preliminaries. We briefly describe our model and refer to [10] (see also [9]) for further details concerning the thermodynamics of materials with internal parameters. In the present context, two forms of heat transmission - diffusive propagation at high temperatures and wavelike propagation at low temperatures - are separated by a phase transition at a critical temperature, $\vartheta_{\lambda}>0$. At temperatures above $\vartheta_{\lambda}$, we

* Received by the editors July 24, 2004; accepted for publication (in revised form) March 2, 2006; published electronically July 17, 2006.

http://www.siam.org/journals/siap/66-5/61220.html

${ }^{\dagger}$ Department of Mathematics and Computer Science, Loyola University, New Orleans, LA 70118 (saxton@loyno.edu). The work of this author was partially supported by NSF grant DMS-0104508.

${ }^{\ddagger}$ Department of Mathematics, University of New Orleans, New Orleans, LA 70148 (rsaxton@ math.uno.edu). The work of this author was partially supported by NSF grant DMS-0104489. 
employ the equations for heat flow through a one-dimensional rigid solid, consisting of balance of energy and Fourier's law,

$$
\begin{gathered}
\varepsilon(\vartheta)_{t}+q_{x}=0, \\
q=-k(\vartheta) \vartheta_{x},
\end{gathered}
$$

where $\varepsilon(\vartheta)$ represents internal energy, $\varepsilon^{\prime}(\vartheta)=c_{v}(\vartheta)$ is the specific heat at constant volume, $q$ denotes heat flux, and $k(\vartheta)$ is the heat conductivity.

At temperatures between $\vartheta=0$ (absolute) and $\vartheta=\vartheta_{\lambda}$, experimental results indicate that the constitutive description of $q$ given by (2.2) is inadequate [2]. This then requires the use of an extended form of thermodynamics, in which we employ an internal parameter, $p$, to appropriately model observations. The internal parameter satisfies a particular form of evolution equation (see [10], [9]), and heat flow is described below $\vartheta_{\lambda}$ by the equations

$$
\begin{gathered}
\varepsilon(\vartheta)_{t}+q_{x}=0, \\
p_{t}=g_{1}(\vartheta) \vartheta_{x}+g_{2}(\vartheta) p, \\
q=-\alpha(\vartheta) p,
\end{gathered}
$$

where $g_{1}(\vartheta) \geq 0 \geq g_{2}(\vartheta)$ and $\alpha(\vartheta) \geq 0$ are material functions. The second law of thermodynamics imposes the restriction that $\alpha(\vartheta)=\psi_{20} \vartheta^{2} g_{1}(\vartheta)$, where the constant $\psi_{20}>0$ comes from the Helmholtz free energy, $\psi$, which has the form $\psi=\psi_{1}(\vartheta)+$ $\frac{1}{2} \psi_{20} \vartheta p^{2}$. While satisfying the second law of thermodynamics, the model has internal energy depending only on temperature [9]. In effect, (2.3)-(2.5) permit $q$ to depend on the history of the temperature gradient.

The following constitutive relations will be used for $g_{1}$ and $g_{2}$ (see [10]):

$$
\begin{array}{ll}
g_{1}(\vartheta)=g_{10}(\vartheta)\left(\vartheta_{\lambda}-\vartheta\right)^{r}, & g_{10}(\vartheta)>0, \\
g_{2}(\vartheta)=g_{20}(\vartheta)\left(\vartheta_{\lambda}-\vartheta\right)^{2 r}, & g_{20}(\vartheta)<0,
\end{array}
$$

where $0 \leq \vartheta \leq \vartheta_{\lambda}$. Here $g_{10}, g_{20} \in C\left[0, \vartheta_{\lambda}\right]$ and $r \in(0,1)$. The form of these functions can be derived from experimental data on the wave speed, $U_{E}(\vartheta)$, the heat conductivity, $K(\vartheta)$, and the specific heat, $c_{v}(\vartheta)$, as we now describe.

The characteristic equation for $(2.3)-(2.5)$ is given by

$$
c_{v}(\vartheta) \lambda^{2}+\lambda \alpha^{\prime}(\vartheta) p-\alpha(\vartheta) g_{1}(\vartheta)=0 .
$$

If one considers waves propagating into an undisturbed state $\vartheta=$ constant, $p=0$ $(q=0)$, this provides an expression for $U_{E}$,

$$
\lambda^{2}=U_{E}^{2} \equiv \frac{\alpha(\vartheta) g_{1}(\vartheta)}{c_{v}(\vartheta)}=\psi_{20} \vartheta^{2} \frac{g_{1}^{2}(\vartheta)}{c_{v}(\vartheta)} .
$$

Given experimental measurements of $U_{E}(\vartheta)$ and $c_{v}(\vartheta)$, this specifies $g_{1}(\vartheta)$. It is observed that second sound is a decreasing function of temperature, which we allow to reach zero [1] at $\vartheta=\vartheta_{\lambda}$ (cf. (2.6)). 
In order to find $g_{2}(\vartheta)$, we use measurements of heat conductivity made in nearstationary states, for which fast processes are considered to be minor, $p_{t} \approx 0 .{ }^{1}$ In this case, the difference between solutions to (2.3)-(2.5) and the diffusion equation

$$
\varepsilon(\vartheta)_{t}-\left(K(\vartheta) \vartheta_{x}\right)_{x}=0,
$$

where $K(\vartheta)=-\frac{\alpha(\vartheta) g_{1}(\vartheta)}{g_{2}(\vartheta)}=-\frac{U_{E}^{2}(\vartheta) c_{v}(\vartheta)}{g_{2}(\vartheta)}>0$, becomes small. Requiring $K(\vartheta)$ to remain finite as $\vartheta \rightarrow \vartheta_{\lambda}$ - then leads to the form of $g_{2}(\vartheta)$ in (2.7).

The behavior of the specific heats of $\mathrm{Bi}$ and $\mathrm{NaF}$ is typically considered to be continuous in temperature, and we will assume this here, with

$$
c_{v}(\vartheta) \sim c_{\lambda}, \quad\left|\vartheta_{\lambda}-\vartheta\right| \ll 1, \quad c_{\lambda}>0,
$$

where " $\sim$ " denotes leading order behavior. Otherwise $c_{v}$ is considered to be described by a continuous function which obeys Debye's law, $c_{v}(\vartheta) \sim \vartheta^{3}$, as $\vartheta \rightarrow 0$.

Particular forms of (2.6) and (2.7) chosen to fit available data for crystals of high purity $\mathrm{NaF}$ and $\mathrm{Bi}$ can be found in [10]. Together with (2.11), these result in $U_{E}$ having the general form

$$
U_{E}^{2}(\vartheta) \sim U_{0}^{2}(\vartheta)\left(\vartheta_{\lambda}-\vartheta\right)^{2 r}
$$

for $\vartheta \leq \vartheta_{\lambda}$, with $U_{0}$, a continuous function of $\vartheta$, found experimentally.

For convenience, we introduce the following change of variables:

$$
e=\varepsilon(\vartheta)-\varepsilon\left(\vartheta_{\lambda}\right), \quad \vartheta=\varepsilon^{-1}\left(e+\varepsilon_{\lambda}\right), \quad \text { and } \varepsilon_{\lambda}=\varepsilon\left(\vartheta_{\lambda}\right) .
$$

We may rewrite (2.1), (2.2) in terms of $e>0$ and $q$ as

$$
\begin{gathered}
e_{t}+q_{x}=0, \\
q=-d(e) e_{x},
\end{gathered}
$$

where $d(e)=k(\vartheta) / c_{v}(\vartheta), k(\vartheta)>0$. When $e<0$, equations (2.3), (2.4) become

$$
q_{t}+\frac{h^{\prime}(e)}{h(e)} q q_{x}=f(e)\left\{q+D(e) e_{x}\right\}
$$

where ${ }^{2}$

$$
f(e)=g_{2}(\vartheta), \quad h(e)=\alpha(\vartheta), \quad D(e)=\frac{K(\vartheta)}{c_{\vartheta}(\vartheta)}
$$

\footnotetext{
${ }^{1}$ In this limit, however, thermal waves do not necessarily propagate at the speed dictated by the qualitatively approximate parabolic equation (2.10), but still at a characteristic velocity, $\lambda$, given by (2.9). We also distinguish $p_{t} \approx 0$ (for which $g_{1}(\vartheta), g_{2}(\vartheta) \neq 0$ ) from $p_{t}=0$ (where $\left.g_{1}(\vartheta)=g_{2}(\vartheta)=0\right)$. The former is an assumption concerning the dynamics, such as the time asymptotic behavior which may arise due to damping (for instance, as seen in [4], and which still preserves hyperbolic features such as finite speed of propagation) in the original system. In the latter case, $\vartheta$ is assumed to have reached the transition temperature, $\vartheta=\vartheta_{\lambda}$.

${ }^{2}$ For physical reasons [2], we will here assume that $k\left(\vartheta_{\lambda}\right)=K\left(\vartheta_{\lambda}\right)$ (where $q=-K(\vartheta) \vartheta_{x}=$ $\left.-D(e) e_{x}\right)$, which occurs in the limit $p_{t} \approx 0$ leading to $(2.10) . K(\vartheta)$ is sometimes known as the quasistatic heat conductivity.
} 
Continuous initial data, $e_{0}(x)$ or $\vartheta_{0}(x)$, will be defined such that

$$
e(\vartheta(x, 0))=e_{0}(x), \quad e_{0}(0)=0,
$$

with

$$
x e_{0}(x)>0 \quad \text { for } x \neq 0,
$$

where

$$
\vartheta(x, 0)=\vartheta_{0}(x), \quad \vartheta_{0}(0)=\vartheta_{\lambda} .
$$

Given the observed sharp decay in the speed of heat pulse propagation, nonlinear effects are involved. Since the system (2.16), (2.17) is quasilinear and hyperbolic (cf. (2.3)-(2.5)), it is possible to account for this decay, but it also becomes possible for shocks to form in finite times [7], [8], [10] in temperatures below $\vartheta_{\lambda}$. The present analysis examines the situation under which solutions taking values at temperatures on both sides of $\vartheta_{\lambda}$ should remain smooth. As such, it can be regarded as a first step in analyzing experiments using large amplitude temperature pulses crossing into phases that involve dissipation.

3. Properties of phase transitions. Let $\Gamma$ denote a curve $x=\varphi(t), t \geq 0$, such that $e(\varphi(t), t)=0\left(\vartheta(\varphi(t), t)=\vartheta_{\lambda}\right)$ and let $V \subset \mathbb{R}_{+}^{2}=\left\{(x, t) \in \mathbb{R}^{2}, t \geq 0\right\}$ be a neighborhood of $\Gamma$. Set $V=U_{-} \cup \Gamma \cup U_{+}$, where the regions $U_{-}$and $U_{+}$correspond to $e<0$ and $e>0$, respectively. Heat propagation is then governed by $(2.14),(2.15)$ in $U_{-}$and by $(2.16),(2.17)$ in $U_{+}$.

We denote limits of a function $u$ from the left and right of $\Gamma$, as $x \rightarrow \varphi(t)$, by $u^{-}(t)=u(\varphi(t)-, t)$ and $u^{+}(t)=u(\varphi(t)+, t)$, respectively, and denote the jump of $u$ across $\Gamma$ by $[u](t)=u^{+}(t)-u^{-}(t)$. Let $P C^{1}(Q)$ denote the class of piecewise differentiable functions on $Q \subset \mathbb{R}^{2}$. By assuming $\vartheta \in P C^{1}\left(\mathbb{R}_{+}^{2}\right)$, $e$ becomes continuous across $\Gamma$.

Let $s=\dot{\varphi}$. Using (2.14) and (2.16) together with the jump condition across $\Gamma$,

$$
-s[e]+[q]=0,
$$

demonstrates that $q$ is continuous across $\Gamma$. This allows us to define

$$
q_{b}(t) \equiv q(\varphi(t), t)=-\lim _{x \rightarrow \varphi(t)+}\left(d(e) e_{x}\right)=-k\left(\vartheta_{\lambda}\right) \vartheta_{x}^{+}(t) .
$$

In the following, where we wish to categorize $s$ and to obtain a relationship between $\vartheta_{x}^{+}(t)$ and $\vartheta_{x}^{-}(t), \vartheta$ and $q$ are considered to be smooth in $V \backslash \Gamma$ (at least locally). For convenience, we next set $\psi_{20}=1$.

LEMMA 3.1. Let $\vartheta_{x}^{+}>0$. Then $s \vartheta_{x}^{-}=0$, and $q \in C^{1}(V)$ if $s=0$.

Proof. Let us write (2.17) in the form

$$
q q_{x}=\frac{h(e)}{h^{\prime}(e)}\left(f(e)\left\{q+D(e) e_{x}\right\}-q_{t}\right) .
$$

Using (2.6)-(2.13), (2.18) in $U_{-}$for $e \sim 0\left(\vartheta \sim \vartheta_{\lambda}\right)$ gives

$$
\begin{gathered}
c_{v}(\vartheta) \sim c_{\lambda}, \\
h(e) \sim \vartheta_{\lambda}^{2} g_{10}\left(\vartheta_{\lambda}\right)\left(\frac{1}{c_{\lambda}}|e|\right)^{r},
\end{gathered}
$$




$$
f(e) \sim g_{20}\left(\vartheta_{\lambda}\right)\left(\frac{1}{c_{\lambda}}|e|\right)^{2 r}
$$

and

$$
D(e) \sim-\frac{\left(\vartheta_{\lambda} g_{10}\left(\vartheta_{\lambda}\right)\right)^{2}}{c_{\lambda} g_{20}\left(\vartheta_{\lambda}\right)} .
$$

Thus, since $D(e) e_{x}=D(e) c_{v}(\vartheta) \vartheta_{x},(3.3)$ implies

$$
q q_{x} \sim-\frac{1}{r} e q_{t} \rightarrow 0 \quad \text { as } e \rightarrow 0^{-} .
$$

Using (3.2) for $\vartheta_{x}^{+}>0$ implies that $q_{x}^{-}=0$ and consequently $e_{t}^{-}=0$, by (2.16). The definition of $\Gamma$ implies that $e_{t}^{-}+s e_{x}^{-}=0$, and so $s e_{x}^{-}=0$, whence $s \vartheta_{x}^{-}=0$.

Finally, if $s=0$, the definition of $\Gamma$ also implies that $e_{t}^{+}=0$, in which case (2.14) implies $q_{x}^{+}=0$.

Assuming that solutions depend continuously on the initial data (2.21) locally in time in $V$, we now obtain the following.

Corollary 3.2. Let $\vartheta_{0}^{\prime \pm}=\lim _{x \rightarrow 0 \pm} \vartheta_{0}^{\prime}(x)>0$. Then there exists $\tau>0$ such that $s=0$ for $t \in(0, \tau)$.

Proof. This follows immediately from the lemma, since $\vartheta_{x}^{-}(t), \vartheta_{x}^{+}(t)>0$, through continuous dependence, over some interval $t \in(0, \tau)$.

Remark. Since $\varphi(0)=0$ from (2.19), in this case $\varphi(t) \equiv 0$ for all $t \in(0, \tau)$.

The next results provide a connection between the left and right states of $\Gamma$ and show that the condition $s=0$ is controlled only by initial data corresponding to these states and by the solution to the diffusion equation, (2.14), (2.15), and (2.19). $(0, \tau)$.

Lemma 3.3. Let $\vartheta_{0}^{\prime}, \vartheta_{0}^{+}>0$ and $s=0$. Then $\vartheta_{x}^{+}(t)>0 \Rightarrow \vartheta_{x}^{-}(t)>0, t \in$

Proof. Let $\gamma_{\alpha} \subset U_{-}$denote the line segment $x=\alpha<0, t \in(0, \tau)$, which lies parallel to $\Gamma$. For $\alpha$ sufficiently small, $\left.q\right|_{\gamma_{\alpha}}$ can be bounded above, strictly, by 0 . This can be seen by defining a curve $\gamma_{\beta} \in U_{-} \cup \Gamma$ as follows,

$$
\gamma_{\beta}=\left\{(x, t(x)), x \leq 0: \frac{d t}{d x}=\frac{h(e)}{h^{\prime}(e) q}, t(0)=\beta \geq 0\right\}
$$

and by writing (2.17) in the form

$$
\left.\frac{1}{2} \frac{d q^{2}}{d x}\right|_{\gamma_{\beta}}=\frac{h(e)}{h^{\prime}(e)} f(e)\left\{q+D(e) e_{x}\right\} \equiv \frac{h}{h^{\prime}} \mathcal{M} .
$$

Using (2.7), (2.18), and (3.5), we find that

$$
\left.\frac{h(e)}{h^{\prime}(e)}\right|_{\gamma_{\alpha}} \sim \frac{e}{r}
$$

for $\alpha \sim 0$.

Also, $D(e) e_{x}=K(\vartheta) \vartheta_{\left.x\right|_{\gamma_{\alpha}}} \rightarrow k\left(\vartheta_{\lambda}\right) \vartheta_{x}^{-}$and $q_{\left.\right|_{\gamma_{\alpha}}} \rightarrow q_{b}=-k\left(\vartheta_{\lambda}\right) \vartheta_{x}^{+}$as $\alpha \rightarrow 0$. Since $e_{\left.\right|_{\gamma_{\alpha}}} \rightarrow 0$ as $\alpha \rightarrow 0$, and through (3.6), it follows that as $\alpha \rightarrow 0$,

$$
\mathcal{M}_{\left.\right|_{\gamma_{\alpha}}} \sim g_{20}\left(\vartheta_{\lambda}\right) k\left(\vartheta_{\lambda}\right)\left(\frac{1}{c_{\lambda}}\right)^{2 r}\left(\vartheta_{x}^{-}-\vartheta_{x}^{+}\right)\left(|e|^{2 r}\right)_{\left.\right|_{\gamma_{\alpha}}} \sim 0 .
$$


As a result, by (3.10),

$$
q(x, t)_{\left.\right|_{\gamma_{\beta}}} \sim q_{b}(\beta),
$$

where

$$
\left.\frac{d t}{d x}\right|_{\gamma_{\beta}} \sim \frac{e}{r q} .
$$

Next, the use of (2.16), (2.17), and (3.12) shows that

$$
\left.\left(q_{t}-\frac{r}{e} q e_{t}\right)\right|_{\gamma_{\alpha}} \sim 0
$$

from which a rearrangement and integration give

$$
q(\alpha, t) \sim q(\alpha, 0)\left(\frac{e(\alpha, t)}{e_{0}(\alpha)}\right)^{r} .
$$

Since $\vartheta_{0}^{\prime+}>0$ and $e_{0}(\alpha)<0$, we have, locally for $x<0$ and $t>0$, that $e<0$ and $q \sim q_{b} \sim-k\left(\vartheta_{\lambda}\right) \vartheta_{x}^{+}<0$. Consequently $\left.\frac{d t}{d x}\right|_{\gamma_{\beta}}>0$ by (3.14), and hence there exists some $\beta>0$ such that $q(\alpha, 0) \sim q_{b}(\beta)$, with $\beta \rightarrow 0+$ as $\alpha \rightarrow 0-$. Therefore (3.16) implies

$$
q_{b}(t)=q_{b}(0) \lim _{\alpha \rightarrow 0}\left(\frac{e(\alpha, t)}{e_{0}(\alpha)}\right)^{r} .
$$

Given $\varepsilon^{\prime}(\vartheta)=c_{\vartheta}(\vartheta)$ and (2.11), (2.13), and (3.4), we have

$$
\lim _{\alpha \rightarrow 0} \frac{e(\alpha, t)}{e_{0}(\alpha)}=\lim _{\alpha \rightarrow 0} \frac{\vartheta_{\lambda}-\vartheta(\alpha, t)}{\vartheta_{\lambda}-\vartheta_{0}(\alpha)}=\frac{\vartheta_{x}^{-}(t)}{\vartheta_{0}^{\prime-}} .
$$

Finally, since $q_{b}(t) / q_{b}(0)=\vartheta_{x}^{+}(t) /{\vartheta^{\prime}}_{0}^{+}$, this implies

$$
\frac{\vartheta_{x}^{-}(t)}{\vartheta_{0}^{\prime-}}=\left(\frac{\vartheta_{x}^{+}(t)}{\vartheta_{0}^{\prime+}}\right)^{1 / r},
$$

which leads to the desired conclusion.

TheOREM 3.4. Let $\vartheta_{0}^{\prime-}, \vartheta_{0}^{\prime+}>0$, and $\vartheta_{x}^{+}(t)>0$ for $t \in(0, T)$, where $T>0$. Then $s=0$ for $t \in(0, T)$.

Proof. Suppose that $t^{\sharp}<T$ denotes the first time for which $\vartheta_{x}^{-}(t)=0$, so that $t^{\sharp}>0$ by Corollary 3.1. Using Lemma 3.1 and continuity in $t$ implies $s\left(t^{\sharp}\right)=0$, and thus Lemma 3.2 holds for $t \in\left(0, t^{\sharp}\right)$. Using continuity once again then implies that $\vartheta_{x}^{+}\left(t^{\sharp}\right)=0$, which violates the hypothesis $\vartheta_{x}^{+}(t)>0$ for $t \in(0, T)$. The result follows through contradiction.

4. Smooth solutions in the transcritical region. Next we set up the problem of phase transition in the region $U_{-} \cup \Gamma \cup U_{+}$, where we now extend $U_{+}$to all of $\mathbb{R}_{++}^{2}=\left\{(x, t) \in \mathbb{R}^{2}, t \geq 0, x>\varphi(t)\right\}$ and restrict $U_{-}$to temperatures close to $\vartheta_{\lambda}$. Assuming $\vartheta_{0}^{\prime+}, \vartheta_{0}^{\prime}{ }^{-}>0$ means that we may set $\varphi(t)=0$ for $t>0$, given $\varphi(0)=0$, from the results of the previous section.

The transcritical phase transition problem reduces to solving

$$
e_{t}-\left(d(e) e_{x}\right)_{x}=0
$$


in $U_{+}$, by (2.14) (2.15). In $U_{-}$, equations (2.16), (2.17) become

$$
\begin{gathered}
e_{t}+q_{x}=0, \\
q_{t}+\frac{r q}{e} q_{x}=0,
\end{gathered}
$$

where we have used (3.11) to obtain (4.3). We recall that across $\Gamma$, where now

$$
e(0, t)=0, \quad t>0
$$

both $e$ and $q$ are continuous. Initial data for (4.1)-(4.3) are given by

$$
e(x, 0)=e_{0}(x), \quad \text { with } x e_{0}(x)>0 \text { for } x \neq 0, \quad \text { and } e_{0}(0)=0 .
$$

In $U_{+}, e(x, t)$ is a solution to (4.1) satisfying the Dirichlet boundary condition (4.4), together with (4.5). This solution determines $q_{b}(t)$,

$$
q_{b}(t)=q(0, t)=-\lim _{x \rightarrow 0+}\left(d(e(x, t)) e_{x}(x, t)\right)=-k\left(\vartheta_{\lambda}\right) \vartheta_{x}^{+}(t),
$$

as a function of the initial data $e_{0}(x)$, for $x>0$.

In $U_{-}$, the pair $(e(x, t), q(x, t))$ is a solution to (4.2), (4.3) satisfying (4.4), (4.5), and (4.6).

Eigenvalues of the system (4.2), (4.3) are given by $\lambda_{\alpha}=0<\lambda_{\beta}=\frac{r q}{e}$. Due to the fact that $q$ does not possess initial data, characteristic curves $\gamma_{\alpha}, \gamma_{\beta}$ are parametrized by $t$ and $x$, respectively (see (3.9), (3.14)):

$$
\left.\frac{d x}{d t}\right|_{\gamma_{\alpha}}=0,\left.\quad x(t ; \alpha)\right|_{\gamma_{\alpha}}=\left.x(0 ; \alpha)\right|_{\gamma_{\alpha}}=\alpha<0
$$

and

$$
\left.\frac{d t}{d x}\right|_{\gamma_{\beta}}=\frac{e}{r q},\left.\quad t(x ; \beta)\right|_{\gamma_{\beta}}>0,\left.t(0 ; \beta)\right|_{\gamma_{\beta}}=\beta>0 .
$$

This system has Riemann invariants, $|e|^{r} / q$ and $q$, which satisfy

$$
\left.\frac{|e|^{r}}{q}\right|_{\gamma_{\alpha}}=\text { constant }
$$

and

$$
q_{\left.\right|_{\beta}}=\text { constant. }
$$

In order to examine when solutions in $U_{-}$remain smooth, we use the following results.

Lemma 4.1. Let $(e, q) \in C^{1}\left(U_{-}\right)$, and suppose that there are constants $\delta>$ $0, \alpha_{0}<0$ such that $e_{0}(\alpha)<-\delta$ for $\alpha<\alpha_{0}$ and $q_{b}(\beta)>-1 / \delta$ for $\beta>0$. Then for each $\beta$ small enough, there is a unique characteristic, $\gamma_{\beta}$, connecting $\Gamma$ with $\mathbb{R}_{-}=$ $\{(x, t): x<0, t=0\}$.

Proof. Consider a region $\mathcal{W} \subset U_{-}$bounded to the left and right by the line $x=\alpha<0$ and by $\Gamma$, and to the top and bottom by the characteristic $\gamma_{\beta}$ and by $\mathbb{R}_{-}$. 


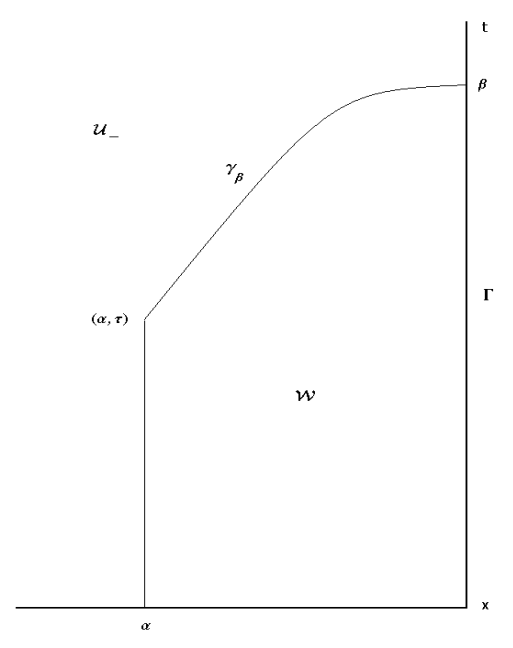

FIG. 4.1.

Let $\gamma_{\beta}$ meet the line $x=\alpha$ at $(x, t)=(\alpha, \tau)$, where $\tau=\tau(\alpha, \beta) \leq \beta$ by (4.8) (see Figure 4.1). We assume in the following that $\gamma_{\beta}$ is entirely contained inside $U_{-}$.

Applying the divergence theorem to (4.2) on $\mathcal{W}$, and using (4.10), provides the relation

$$
\int_{\alpha}^{0} e_{0}(x) d x=\int_{0}^{\beta} q_{b}(t) d t-(1-r) \beta q_{b}(\beta)+\tau(1-r) q_{b}(\beta)-\int_{0}^{\tau} q(t, \alpha) d t .
$$

Fixing $\beta$ in (4.11) and differentiating with respect to $\alpha$ then gives

$$
\begin{aligned}
-e_{0}(\alpha) & =\left((1-r) q_{b}(\beta)-q(\tau, \alpha)\right) \frac{\partial \tau}{\partial \alpha} \\
& =-r q_{b}(\beta) \frac{\partial \tau}{\partial \alpha} .
\end{aligned}
$$

So $\frac{\partial \tau}{\partial \alpha}=\frac{e_{0}(\alpha)}{r q_{b}(\beta)}>\delta^{2} / r$ for $\alpha<\alpha_{0}$, which means that $\tau(\alpha, \beta)$ is bounded below, for fixed $\beta$, by a uniformly increasing function of $\alpha$. Since $\tau(0, \beta)=\beta>0$, it follows that $\tau$ must reduce to zero as $\alpha$ decreases from $\alpha=0$, at which point $\gamma_{\beta}$ meets $\mathbb{R}_{-}$.

We note that if $\gamma_{\beta}$ connects $\Gamma$ to $\mathbb{R}_{-}$, then (4.11) reduces to

$$
\int_{\alpha}^{0} e_{0}(x) d x=\int_{0}^{\beta} q_{b}(t) d t-(1-r) \beta q_{b}(\beta),
$$

which gives a functional relation between $\alpha$ and $\beta$. In particular, differentiating with respect to $\alpha$,

$$
-e_{0}(\alpha) \frac{d \alpha}{d \beta}=r q_{b}(\beta)-(1-r) \beta q_{b}^{\prime}(\beta)
$$

shows that $\frac{d \alpha}{d \beta}<0$ if $r q_{b}(\beta)-(1-r) \beta q_{b}^{\prime}(\beta)<0$. Thus, at least for small $\beta>0$, one finds that $\frac{d \alpha}{d \beta}<0$, since $q_{b}(0)<0$ due to our assumption $\vartheta_{0}^{\prime+}>0$. 


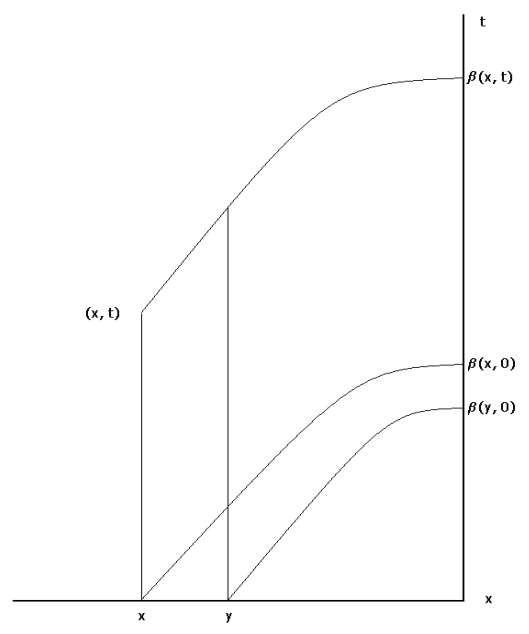

FIG. 4.2 .

We will use the notation $\beta=\beta(\alpha, \tau)$ to denote the time of intersection of the characteristic curve going through $(x, t)=(\alpha, \tau)$, with $\Gamma$, i.e., $\beta=\beta(\alpha, \tau(\alpha, \beta)), \alpha<0$. The inversion is possible whenever $\frac{\partial \tau}{\partial \beta}>0$, which is a subject of the following results.

Lemma 4.2. Let $(e, q) \in C^{1}\left(U_{-}\right), q_{b}(\beta)<0$, and $q_{b}^{\prime}(\beta) \neq 0$. Then $\frac{\partial t}{\partial \beta}(x, \beta)>0$, and if $\frac{\partial t}{\partial \beta} \rightarrow 0$ as $x \rightarrow x^{*}<0$, then $\left|q_{t}(x, t(x, \beta))\right| \rightarrow \infty$.

Proof. By (4.10), we have that

$$
q(x, t(x, \beta))=q_{b}(\beta)
$$

along $\gamma_{\beta}$, and so $q_{\left.\right|_{\gamma_{\beta}}}<0$. Differentiating relation (4.15) in $\beta$ gives

$$
\left(q_{t} t_{\beta}\right)_{\left.\right|_{\gamma_{\beta}}}=q_{t}(0, t(0, \beta))=q_{b}^{\prime}(\beta)
$$

since $t(0, \beta)=\beta$. Thus $\frac{\partial t}{\partial \beta} \rightarrow 0 \Leftrightarrow\left|q_{t}\right|_{\gamma_{\beta}} \rightarrow \infty$.

In the following we take, for simplicity, $U_{-}$to be the vertical strip $\left\{(x, t) \in \mathbb{R}_{+}^{2}\right.$ : $\left.x^{*}<x<0\right\}$ for some $x^{*}<0$. With regard to the theorem, we remark that the two crystalline materials, bismuth and sodium fluoride, have values of the material constant, $r$, lying between zero and one-half [10].

THEOREM 4.3. Let $\beta_{1} \neq \beta_{2}$. Then $\gamma_{\beta_{1}}$ and $\gamma_{\beta_{2}}$ do not intersect in $U_{-}$, provided that one of the following holds:

Case 1. $r \in(0,1)$, and either

(i) $q_{b}^{\prime}(\beta)>0$

or

(ii) $q_{b}^{\prime}(\beta)<0$ and $r q_{b}(\beta)-(1-r) \beta q_{b}^{\prime}(\beta)<0$.

Case 2. $r>1$, and either

(i) $q_{b}^{\prime}(\beta)<0$

or

(ii) $q_{b}^{\prime}(\beta)>0$ and $r q_{b}(\beta)-(1-r) \beta q_{b}^{\prime}(\beta)<0$. 
Case 3. $r=1$.

Proof. Recalling (4.9) and (4.10), for $(x, t) \in U_{-}$,

$$
\frac{|e(x, t)|^{r}}{q_{b}(\beta(x, t))}=\frac{|e(x, t)|^{r}}{q(x, t)}=\frac{\left|e_{0}(x)\right|^{r}}{q(x, 0)}=\frac{\left|e_{0}(x)\right|^{r}}{q_{b}(\beta(x, 0))},
$$

which gives

$$
|e(x, t)|^{r}=\frac{q_{b}(\beta(x, t))}{q_{b}(\beta(x, 0))}\left|e_{0}(x)\right|^{r} .
$$

As a result, (4.8) can be written as

$$
\left.\frac{d t}{d x}\right|_{\gamma_{\beta}}=-\left|q_{b}(\beta(x, t))\right|^{(1-r) / r} \frac{e_{0}(x)}{r\left|q_{b}(\beta(x, 0))\right|^{1 / r}} .
$$

Integrating (4.19) along $\gamma_{\beta}$ and using (4.10) again,

$$
t=t(x, \beta)=\beta-\left|q_{b}(\beta)\right|^{(1-r) / r} \int_{0}^{x} \frac{e_{0}(y)}{r\left|q_{b}(\beta(y, 0))\right|^{1 / r}} d y
$$

with $x *<x<0$ and $0<t<\beta$. Thus, differentiating with respect to $\beta=\beta(x, t)$,

$$
\frac{\partial t}{\partial \beta}=1-\frac{1-r}{r}\left|q_{b}(\beta)\right|^{1 / r-3} q_{b}(\beta) q_{b}^{\prime}(\beta) \int_{0}^{x} \frac{e_{0}(y)}{r\left|q_{b}(\beta(y, 0))\right|^{1 / r}} d y
$$

Case 1(i) of the proof follows since $e_{0}<0, q_{\mathrm{b}}<0$, and characteristics spread with decreasing $x, \frac{\partial t}{\partial \beta}>1$.

In order to establish Case 1(ii), let us first suppose that $\left(x^{*}, t^{*}\right) \in \partial U_{-}$for $x^{*}<0$, and that $t^{*}>0$ is the least time at which solutions may fail to be in $C^{1}$. Next, assume that $\beta_{1}<\beta_{2}$ are such that $\gamma_{\beta_{1}}$ and $\gamma_{\beta_{2}}$ intersect at $\left(x^{*}, t^{*}\right)$, where $\gamma_{\beta_{i}}=\{(x, t): t=$ $\left.t\left(x, \beta_{i}\right)\right\}, i=1,2$. So (4.20) holds for $i=1, i=2$ at $\left(x^{*}, t^{*}\right)$,

$$
t^{*}\left(x^{*}, \beta_{i}\right)=\beta_{i}-\left|q_{b}\left(\beta_{i}\right)\right|^{(1-r) / r} \int_{0}^{x^{*}} \frac{e_{0}(y)}{r\left|q_{b}\left(\beta_{i}(y, 0)\right)\right|^{1 / r}} d y, \quad i=1,2,
$$

where we note that the integral terms are identical for $i=1,2$. Eliminating this term leads to the relation

$$
t^{*}-\beta_{2}=\left(t^{*}-\beta_{1}\right)\left(\frac{q_{b}\left(\beta_{2}\right)}{q_{b}\left(\beta_{1}\right)}\right)^{(1-r) / r},
$$

where $\left.Q \equiv \frac{q_{b}\left(\beta_{2}\right)}{q_{b}\left(\beta_{1}\right)}\right)^{(1-r) / r}>1$ since $q_{b}^{\prime}<0$, by our hypothesis. However, it is easily shown that conditions (ii) imply

$$
1<\mathcal{Q}<\frac{\beta_{2}}{\beta_{1}}
$$

Thus $t^{*}>0$ cannot exist since (4.23), (4.24) imply

$$
t^{*}=\beta_{1} \frac{\beta_{2} / \beta_{1}-Q}{1-Q}<0 .
$$


Cases 2(i) and (ii) can be established as above, and Case 3 follows since (4.20) then implies $t_{\beta} \equiv 1$.

Remark. In addition to $q_{t}$, the terms $e_{t}, q_{x}$, and $e_{x}$ naturally blow up where $\frac{\partial t}{\partial \beta} \rightarrow 0$, under the conditions of Theorem 4.3. This results from $q$ remaining constant on $\gamma_{\beta}$ and $e$ remaining bounded away from zero on $\gamma_{\alpha}$ by (4.10) and (4.9). Equation (4.3) then implies $\left|q_{x}\right| \rightarrow \infty$ as $\left|q_{t}\right| \rightarrow \infty$, and therefore $\left|e_{t}\right| \rightarrow \infty$ by (4.2). On differentiating (4.9) in $\alpha$, the same result follows for $\left|e_{x}\right|$.

Let us finally state some relationships more concisely. For any point $(x, t) \in U_{-}$, the solution $(e(x, t), q(x, t))$ to $(4.2),(4.3)$ subject to (4.4), (4.5), and (4.6) takes the form

$$
\begin{gathered}
q(x, t)=q_{b}(\beta(x, t)) \\
e(x, t)=\left(\frac{q_{b}(\beta(x, t))}{q_{b}(\beta(x, 0))}\right)^{1 / r} e_{0}(x),
\end{gathered}
$$

with

$$
t=\beta(x, t)-\left(\frac{q_{b}(\beta(x, t))}{q_{b}(\beta(x, 0))}\right)^{(1-r) / r} \beta(x, 0),
$$

where $\beta(x, 0)$ is derived from (4.13) (with $\alpha=x$ ) and (4.28) comes from repeated use of (4.20) with $t>0$ and $t=0$ to eliminate the integral term. Using Theorem 4.3, we may invert (4.28) for fixed $x$ in order to obtain $\beta(x, t)$ used in (4.26) and (4.27). Similarly, by using (4.20) in (4.21), one can find $\frac{\partial t}{\partial \beta}$ in terms of $\beta(x, t)$ in the useful form

$$
\frac{\partial t}{\partial \beta}=1-\frac{1-r}{r} \frac{q_{b}^{\prime}(\beta)}{q_{b}(\beta)}(\beta-t) .
$$

5. Some explicit smooth solutions. Having observed the role that heat flux continuity across $\Gamma$ plays in solving (4.2)-(4.3), we now examine the effect on $U_{-}$of having a stationary or self-similar solution to (4.1) in $U_{+}$. The solutions are defined for $U_{-}$lying in the transcritical region.

In the case that the $U_{+}$component of the solution is stationary, i.e., $e_{t}=q_{x}=0$ with $q=-d(e) e_{x}<0$, this implies that $q_{b}(\beta)=q_{b}(0)$ for all $\beta>0$. Since $\left.q\right|_{\gamma_{\beta}}$ remains constant, this means in turn that $q=q_{b}(0)$ in $U_{-}$. Consequently, since $\left.\left(|e|^{r} / q\right)\right|_{\gamma_{\alpha}}$ is also constant, it follows that $e(x, t)=e_{0}(x)$ for arbitrary initial temperature distributions, $e_{0}(x)$, in $U_{-}$. As a result, the solution is everywhere stationary.

In the next case, we choose $U_{+}$to be governed by the linear heat equation $d(e)=1$ (assuming $c_{\lambda}=1$ ), in which we can use, for example, any explicit solution formula for the semi-infinite interval. We set up the following example by means of a self-similar solution, in $U_{+}$, of the form $e(x, t)=e(x / \sqrt{t+1})$ and let this extend to $U_{-}$through $\Gamma$. The solution in $U_{+}$is then represented by

$$
e(x, t)=A \sqrt{\pi} \operatorname{erf}\left(\frac{x}{2 \sqrt{t+1}}\right), \quad A>0,
$$

giving heat flux as

$$
q(x, t)=-\frac{A}{\sqrt{t+1}} e^{-x^{2} / 4(t+1)} \quad \text { with } q_{b}(t)=-\frac{A}{\sqrt{t+1}} .
$$




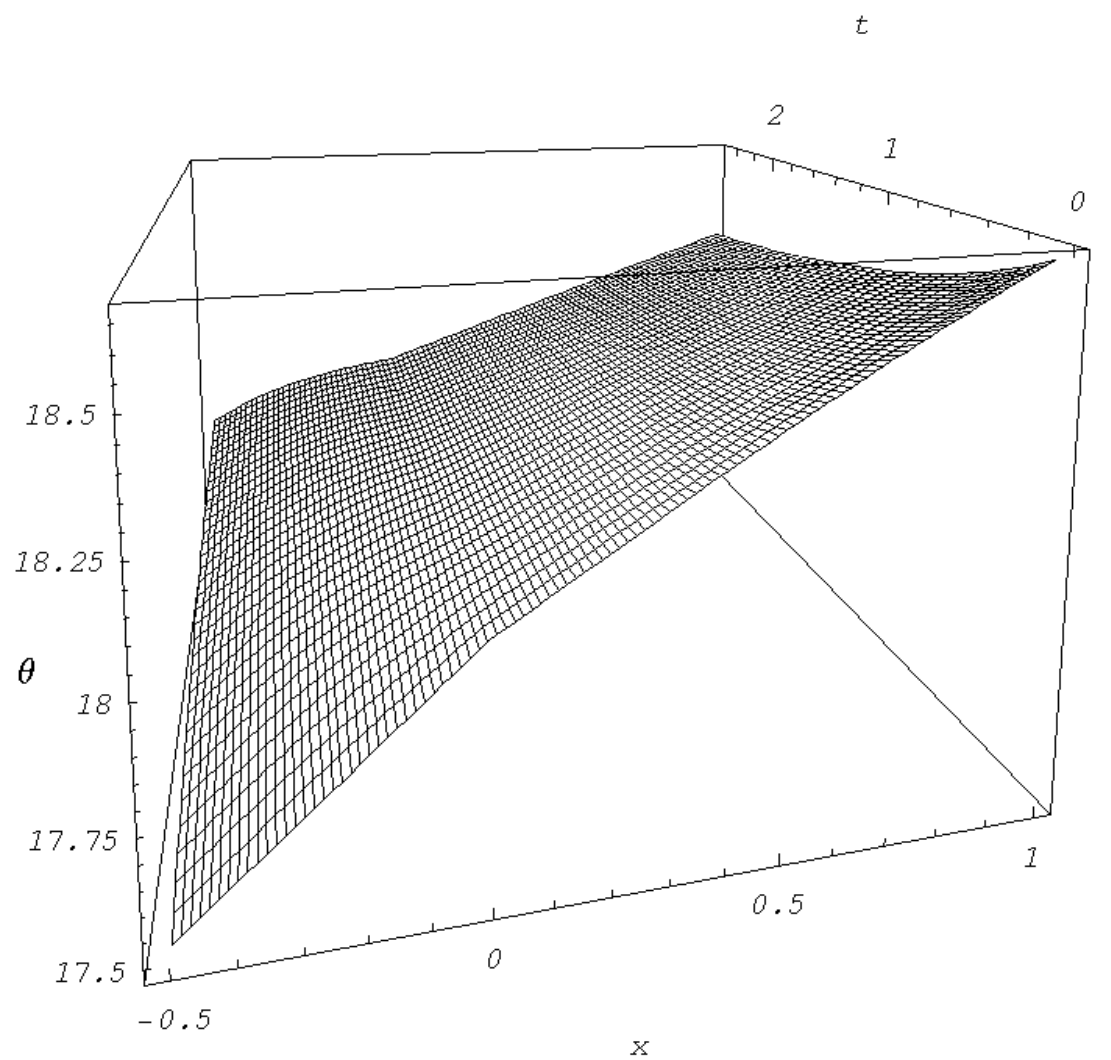

FIG. 5.1. Temperature evolution with $\vartheta_{\lambda}=18 K$, for $0 \leq t \leq 2.5 \mu \mathrm{sec}$. and $-.5 \leq x \leq 1 \mathrm{~cm}$.

Since $q_{b}^{\prime}(t)>0$, Theorem 4.1 guarantees that the characteristics in $U_{-}$do not intersect. We choose as initial data for $e$ in $U_{-}$,

$$
e_{0}(x)=x, \quad x<0,
$$

which is equivalent to $\vartheta_{0}(x)=x+\vartheta_{\lambda}$. The following connection can now be made from (4.13) (with $\alpha=x$ ),

$$
\beta(x, 0)=\left(\frac{B(x)+\sqrt{B(x)^{2}-\left(1-r^{2}\right)}}{1+r}\right)^{2}-1,
$$

where

$$
B(x)=\frac{1}{4 A} x^{2}+1
$$

Equation (4.28) then leads to a relation for $\beta=\beta(x, t)$,

$$
t=\beta-(\beta+1)^{-(1-r) / 2 r}(\beta(x, 0)+1)^{(1-r) / 2 r} \beta(x, 0) .
$$

Taking, as a special case, $r=1 / 3$ in (5.6) gives

$$
\beta(x, t)=\frac{t-1+\sqrt{(1+t)^{2}+4 H(x ; 1 / 3)}}{2},
$$




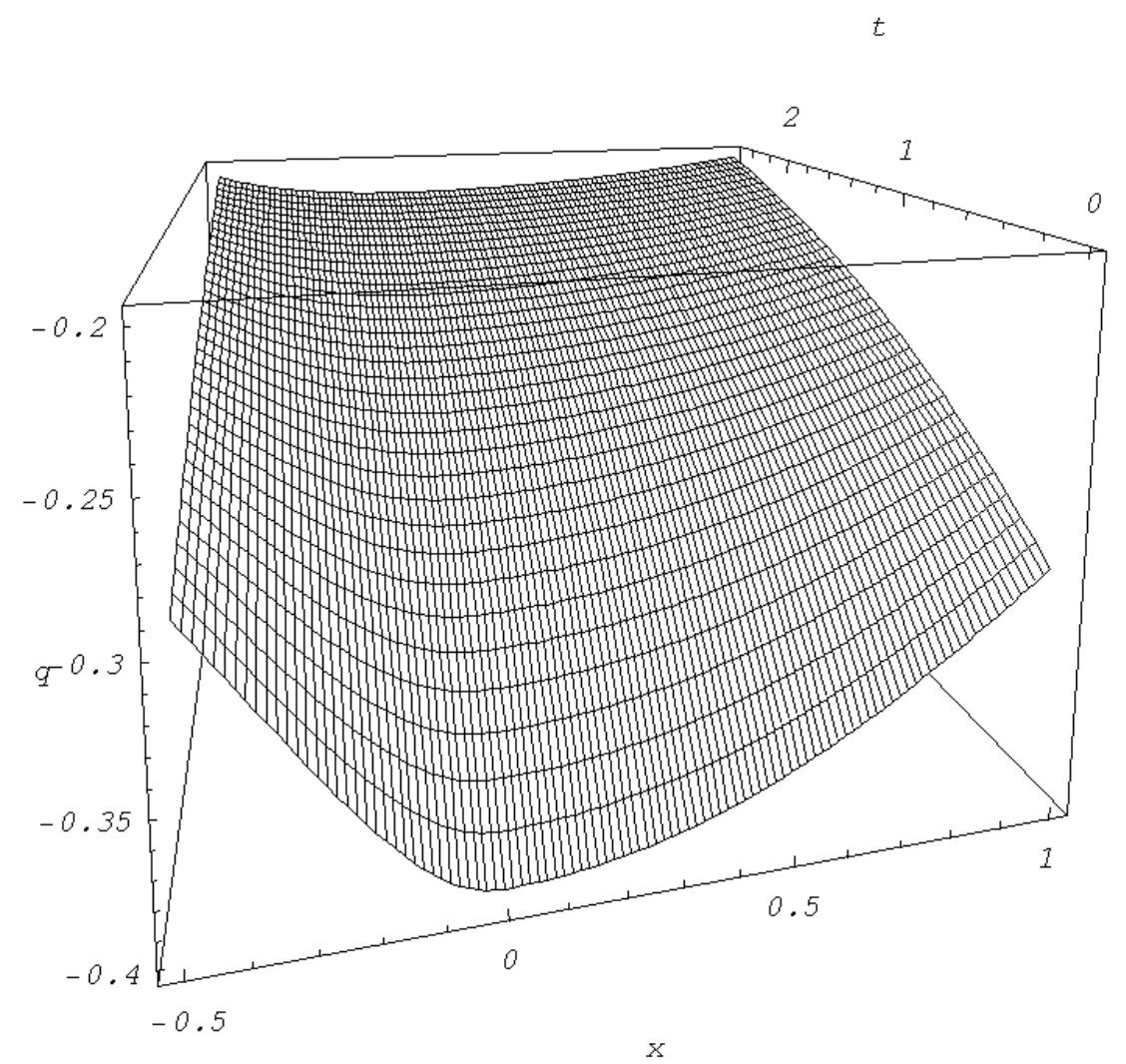

FIG. 5.2. Heat flux evolution with $\vartheta_{\lambda}=18 \mathrm{~K}$, for $0 \leq t \leq 2.5 \mu \mathrm{sec}$., $-.5 \leq x \leq 1 \mathrm{~cm}$, with $-0.4 \leq q<0 \mathrm{~W} / \mathrm{cm}^{2}$.

where we have set

$$
H(x ; r)=(\beta(x, 0)+1)^{(1-r) / 2 r} \beta(x, 0) .
$$

Using (4.26), (4.27) together with (5.2), (5.3), (5.4), and (5.7) finally gives a solution for $(e, q)$ in $U_{-}$,

$$
\begin{gathered}
e(x, t)=\left(\frac{3 \sqrt{2}}{4}\right)^{3}\left(\frac{B(x)+\sqrt{B(x)^{2}-8 / 9}}{\sqrt{t+1+\sqrt{(1+t)^{2}+4 H(x ; 1 / 3)}}}\right)^{3} e_{0}(x), \\
q(x, t)=-\sqrt{2} \frac{A}{\sqrt{t+1+\sqrt{(1+t)^{2}+4 H(x ; 1 / 3)}}},
\end{gathered}
$$

where, from (2.11), (2.13) and the definition $\varepsilon^{\prime}(\vartheta)=c_{v}(\vartheta)$,

$$
\vartheta(x, t)=\vartheta_{\lambda}+e(x, t)
$$

Figures 5.1 and 5.2 illustrate the behavior of $\vartheta(x, t)$ and $q(x, t)$ in $U_{-} \cup \Gamma \cup U_{+}$, using (5.1), (5.2), and (5.9)-(5.11) with $A=2 / 5$. 
One can see in Figure 5.2 that, despite the temperature gradient being initially larger to the left of the phase transition at $x=0$ than to the right (see Figure 5.1), $q$ is nevertheless greater there than at the transition itself. This illustrates distinctly "nonFourier" behavior in $U_{-}$. It is also possible to observe in Figure 5.1 the derivative discontinuity in $\vartheta$ at $x=0$ changing from "concave down" to "concave up" as time progresses. From (3.19), with $r=1 / 3$, this change occurs when $\vartheta_{x}^{+}(t)=\left(\vartheta_{0}^{\prime+}\right)^{3} /\left(\vartheta_{0}^{\prime-}\right)^{1 / 2}$, or at about $t=1$. With $q_{b}^{\prime}(\beta)>0$ and $r<1$, as in the present case, it is easy to check that such a concavity change can occur only if $0<\vartheta_{0}^{\prime+}<\vartheta_{0}^{\prime-}$.

\section{REFERENCES}

[1] H. Beck And R. Beck, Heat-pulse propagation in dielectric solids, Phys. Rev. B, 8 (1973), pp. 1669-1679.

[2] H. E. JaCKSON AND C. T. WALKer, Thermal conductivity, second sound, and phonon-phonon interactions in NaF, Phys. Rev. B, 3 (1971), pp. 1428-1439.

[3] H. E. Jackson, C. T. Walker, and T. F. Mcnelly, Second sound in NaF, Phys. Rev. Lett., 25 (1970), pp. 26-28.

[4] H. Li And K. Saxton, Asymptotic behavior of solutions to quasilinear hyperbolic equations with nonlinear damping, Quart. Appl. Math., 61 (2003), pp. 295-313.

[5] T. F. Mcnelly, S. J. Rogers, D. J. Chamin, R. J. Rollefson, W. M. Goubau, G. E. Schmidt, J. A. Krumhansi, and R. O. Pohl, Heat pulses in NaF: Onset of second sound, Phys. Rev. Lett., 24 (1970), pp. 100-102.

[6] V. Narayanamurti and R. C. Dynes, Observation of second sound in bismuth, Phys. Rev. Lett., 28 (1972), pp. 1461-1465.

[7] T. Ruggeri, A. Muracchini, And L. Seccia, Shock waves and second sound in a rigid heat conductor: A critical temperature for NaF and Bi, Phys. Rev. Lett., 64 (1990), pp. 26402643.

[8] T. Ruggeri, A. Muracchini, And L. Seccia, Second sound and characteristic temperature in solids, Phys. Rev. B, 54 (1996), pp. 332-339.

[9] K. Saxton, R. Saxton, And W. Kosinski, On second sound at the critical temperature, Quart. Appl. Math., 57 (1999), pp. 723-740.

[10] K. Saxton And R. Saxton, Nonlinearity and memory effects in low temperature heat propagation, Arch. Mech., 52 (2000), pp. 127-142. 